\section{Frische Hofmilch als Immuntherapeutikum}

Kinder, die auf einem Bauernhof aufwachsen, sind einer Studie zufolge besser vor Asthma - auch der nicht-allergischen Form - geschützt als kleine Städter. Zwei internationale Forscherteams haben herausgefunden, warum das so ist.

7 ahlreiche Studien haben nahegelegt, dass Bauerhöfe ein bevorzugtes Umfeld darstellen, wenn man Kinder vor Allergien schützen will. Aber beschränkt sich der protektive Effekt nur auf die Atopie? Ein internationales Forscherteam hat die Auswirkungen des Landlebens auf die Funktion der Atemwege bei Kindern zwischen sechs und zwölf Jahren studiert [1]. An den „GABRIEL Advanced Studies" waren über 8.000 allergische und nicht-allergische Kinder aus Österreich, Süddeutschland und der Schweiz beteiligt, die entweder auf einem Bauernhof aufwuchsen oder häufig Kontakt zu einem landwirtschaftlichen Betrieb hatten. Kinder ohne solche Kontakte dienten als Kontrollgruppe. Neben Allergien lag der Fokus auf asthmatypischen Atemnebengeräuschen, vor allem dem Giemen (,Wheezing“), sowie der objektiven Lungenfunktion, gemessen mittels Spirometrie.

Untersucht wurden zunächst spezifische Sensibilisierungen gegen Katzenhaare, Roggen, Gräser, Birkenpollen und Beifuß. Das Risiko einer atopischen Sensibilisierung war bei den Bauernkinder insgesamt deutlich niedriger (Odds Ratio [OR]: 0,51; 95\%iges-Konfidenzintervall $[\mathrm{KI}]: 0,45-0,58)$. Dabei waren sowohl das Ausmaß der Sensibilisierung als auch der Gesamt-IgE-Spiegel klar mit Bauernhofkontakten assoziiert.

Auch bei den Atembeschwerden waren die Hofbewohner klar im Vorteil: Das Relative Risiko für Giemen lag bei $0,78 \mathrm{im}$ Vergleich zur Kontrollgruppe, allerdings nur für eine transiente Form, die vor dem dritten Lebensjahr aufgetreten und dann wieder verschwunden war. Für später aufgetretene Formen waren die Ergebnisse heterogen: Hier fand sich ein deutlich gesenktes Risiko mit einer OR von 0,45 , dies galt aber wiederum nur für Nicht-Allergiker unter den Bauernkindern.

Entgegen den Erwartungen hatte der Kontakt zu Tieren und Ställen jedoch generell weder Auswirkungen auf die Ergebnisse der Spirometrie (FEV1/FVC).
Deutlich war dagegen der Einfluss auf einen anderen Parameter, und zwar speziell für die Gruppe der Allergiker, die auf einem Hof aufwuchsen. Sie hatten deutlich geringere Konzentrationen von Stickoxiden in der Ausatemluft (Fraction of Exhaled Nitric Oxide, FENO, OR: 0,74), ohne dass sich jedoch die Lungenfunktion eindeutig verbessert hätte.

Wie die Studienautoren um Dr. Oliver Fuchs vom Inselspital der Universität Bern spekulieren, scheint eine inverse Assoziation des Bauernhoflebens mit dem Grad der atopischen Sensibilisierung zu existieren; die niedrigen FENO-Werte sprechen dafür. Ein gemeinsamer Nenner für die Effekte sowohl auf die transiente als auch auf die nicht-allergische Wheezing-Symptomatik bestehe möglicherweise in dem Schutz vor Atemwegsinfektionen, so Fuchs. Hier spielt unter Umständen die angeborene Immunantwort eine entscheidende Rolle.

Diesem Zusammenhang sind die Autoren der PASTURE-Studie weiter nachgegangen [2]. Das Studienkollektiv bestand aus 1.133 schwangeren Frauen aus ländlichen Gegenden in Österreich, Finnland, Frankreich, Deutschland und der Schweiz. 530 lebten auf einem Bauernhof. Bei allen Teilnehmerinnen wurden mRNA-Analysen des Nabelschnurbluts durchgeführt, außerdem untersuchte man Blutproben der Kinder, wenn diese ein Jahr alt waren.

Bei den Bäuerinnen war es offenbar zu einer deutlich erhöhten Expression von bestimmten Rezeptorgenen gekommen. Vor allem die Gene, die für die Toll-likeRezeptoren TLR 7 und 8 kodieren, waren mit einer aGMR (,adjusted geometric means ratio") von 1,15 signifikant hochreguliert (95\%-KI: 1,02-1,30; $\mathrm{p}=$ 0,021). Als Bestandteile der angeborenen Immunität erkennen die TLR bestimmte pathogenassoziierte Molekülmuster.

Positive Assoziationen mit spezifischen Genexpressionsmustern fanden sich in erster Linie für das regelmäßige Trinken unpasteurisierter Milch frisch

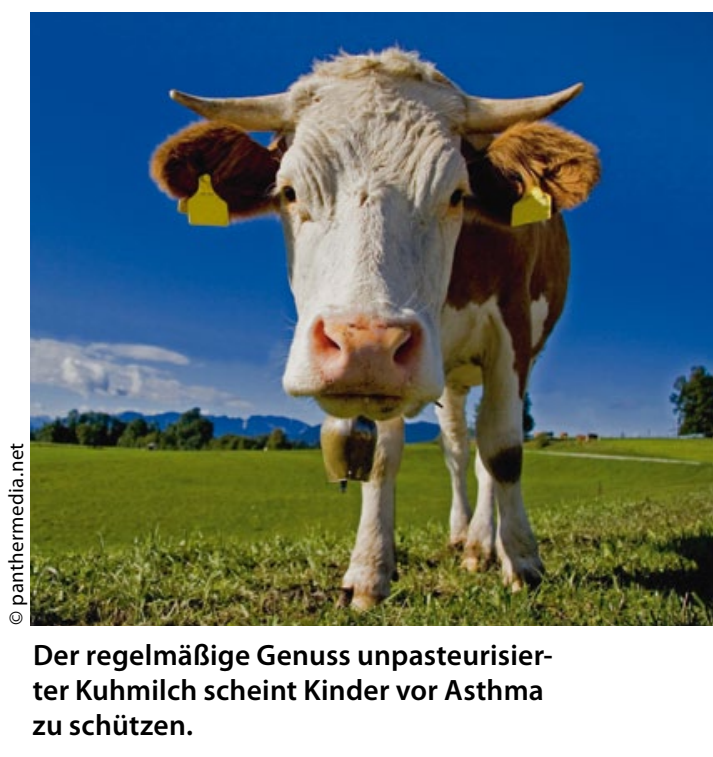

von der Kuh. Dies galt nicht nur für die Mütter, sondern auch für deren Nachwuchs: Kinder, die im ersten Lebensjahr naturbelassene Milch bekommen hatten, zeigten im Vergleich zur Kontrollgruppe eine deutlich stärkere mRNA-Expression für TLR 4, 5 und 6. Naturgemäß hatten vor allem die Bauernkinder Hofmilch erhalten ( 29 vs. $4,5 \%$ ).

Fazit: Das Leben auf einem Bauernhof schützt Kinder vor asthmaspezifischen Beschwerden wie dem sogenannten "Wheezing", und zwar unabhängig von einer allergischen Disposition. Dieser Effekt lässt sich jedoch nicht mit einem vergrößerten Atemwegsvolumen erklären. Vielmehr scheinen Faktoren der angeborenen Immunität, insbesondere das Toll-like-Rezeptorsystem, eine Rolle zu spielen. Dieses wird bei entsprechender Exposition hochreguliert und schützt so möglicherweise vor Atemwegsinfektionen. Der entscheidende Faktor ist dabei offenbar nicht der bloße Kontakt der Kinder zu Tieren und Ställen, sondern vielmehr das regelmäßige Trinken frischer, nicht-pasteurisierter Milch.

Dr. Elke Oberhofer

1. Fuchs $\mathrm{O}$ et al. Farming environments and childhood atopy, wheeze, lung function, and exhaled nitric oxide. J Allergy Clin Immunol 2012;130: 382-8

2. Loss $\mathrm{G}$ et al. Prenatal and early-life exposures alter expression of innate immunity genes: The PASTURE cohort study. J Allergy Clin Immunol 2012; 130: 523-30 